\title{
Semiología nutricional: el mejor camino para la seguridad diagnóstica y terapéutica
}

\author{
Nutritional semiology: The best way for diagnostic and therapeutic safety \\ Semiologia nutricional: o melhor caminho para a segurança de diagnostico e terapêutica
}

\author{
Juan Carlos de la Cruz Castillo Pineda1*, Marthe Alice Chantal Bricard González², \\ Sara Alejandra Díaz Tena ${ }^{3}$, Isabel Guadalupe Calvo Higuera ${ }^{4}$ \\ Recibido: 15 de julio de 2020. Aceptado para publicación: 10 de octubre de 2020 \\ Publicado primero en línea: 24 de octubre de 2020 \\ https://doi.org/10.35454/rncm.v4n1.177
}

\section{Resumen}

Los cambios biomoleculares se expresan en el organismo a través de signos y síntomas, muchos de ellos tan objetivos que pueden ser observados por el médico o nutricionista, pero otros requieren estudios paraclínicos para ser evaluados en forma adecuada a través del acto clínico donde científicamente se obtienen datos para ser integrados en síndromes que serán la base del diagnóstico clínico y proponer luego la meta y plan terapéutico médico o dietético.

Diversas organizaciones de salud han destacado la importancia de unificar criterios para el diagnóstico de la desnutrición y obesidad haciendo énfasis sobre todo en el cambio del peso, cambio en la ingesta y el grado de inflamación que solo expresa riesgo. El abordaje clínico precisa el grado de afectación integrando signos, síntomas clínicos, bioquímicos e imagenológicos que proporcionan certeza al tratamiento. En este articulo se hace la revisión de las formas de abordar algunos problemas clínicos que tienen que ver con el apetito y el funcionamiento del aparato digestivo que son la vía de entrada para una adecuada alimentación, y una correlación de la disbiosis intestinal.

Palabras clave: semiología, apetito, saciedad, enfermedades gastrointestinales.

\section{Summary}

In the body, biomolecular changes are expressed through signs and symptoms, many of them so evident they can readily be observed by a doctor or nutritionist, but others require additional diagnostic studies to be adequately evaluated during the clinical evaluation of the patient, during which data are scientifically obtained to be integrated into syndromes that will ultimately become the basis of clinical diagnosis, and will also serve to propose a goal and medical or dietary therapeutic plan.

Various health organizations have highlighted the importance of unifying diagnostic criteria for malnutrition and obesity, making special emphasis on weight and intake changes, and in the degree of inflammation that only expresses risk. The clinical approach requires determining the degree of involvement by integrating clinical, biochemical and imaging signs and symptoms that provide certainty to the treatment. In this article, we review ways to addressing some of the clinical problems related to appetite and the function of the digestive system, which are the entry point for adequate nutrition, and their link to intestinal dysbiosis.

Keywords: Semiology; Appetite; Satiation Gastrointestinal diseases.

\section{Resumo}

As alterações biomoleculares são expressas no organismo através de sinais e sintomas, muitos deles tão objetivos que podem ser observados pelo médico ou nutricionista, mas outros requerem estudos paraclínicos para serem avaliados de forma adequada através de ato clinico onde cientificamente se obtêm dados para serem integrados em síndromas que serão a base do diagnóstico clinico e propor logo a meta e plano terapêutico médico e dietético.

Várias organizações de saúde destacaram a importância de unificar critérios para o diagnóstico da desnutrição e obesidade, fazendo enfase sobretudo na alteração de peso, na mudança da ingestão e o grau de inflamação que só expressa risco. A abordagem clínica requer o grau de envolvimento, integrando sinais, sintomas clínicos, bioquímicos e de imagem que garantem segurança ao tratamento. Neste artigo se faz uma revisão das formas de abordar de alguns problemas clínicos relacionados com o apetite e o funcionamento do sistema digestivo, que é o caminho de entrada para uma dieta adequada e uma correlação da disbiose intestinal.

Palavras-chave: semiologia, apetite, saciedade, doenças gastrointestinais.
1. Universidad Michoacana de San Nicolás de Hidalgo, Michoacan, México

2 Universidad Anáhuac campus norte, Ciudad de México, México.
3 Universidad Latina de América, Ciudad de México, México.

4 Hospital General de Tijuana, Tijuana, México.

*Correspondencia: Juan Carlos de la Cruz Castillo Pineda castillomorelia@gmail.com 
"En el acto de comer, el placer está tan mezclado con la necesidad que no sabemos qué parte corresponde a cada uno." San Gregorio

Los seres humanos son metazoarios heterótrofos que para sobrevivir dependen de la adecuada armonía con un ambiente en cambio permanente, que le obliga a activar mecanismos de constante adaptación como la carencia o el exceso de alimento, la exposición a compuestos químicos altamente industrializados, que son un verdadero reto para el metabolismo, propiciando ajustes bioquímicos importantes que desencadenan disfunción, inflamación y enfermedades ${ }^{(1)}$.

Estos cambios biomoleculares tienen expresión en los diferentes aparatos y sistemas del organismo. Algunos muy evidentes como la desnutrición marasmática o la obesidad mórbida, pero no siempre es así. Estos cambios, con frecuencia, no son detectados por el sujeto o no les da importancia, y solo se atiende cuando aparece en el horizonte clínico. Es así como el enfermo solicita atención del personal de salud (médico, nutricionista, enfermera, etc.), quienes enfrentan una de las experiencias más apasionantes del ser humano: el acto clínico. En este, el paciente busca solucionar un problema no resuelto, y con frecuencia, espera un resultado mágico y rápido ${ }^{(2,3)}$. Algunas veces acude lleno de preguntas dispersas o influidas por la búsqueda en internet, que con frecuencia lo confunde más, pero otras, llega en silencio o es incapaz de mantener una comunicación verbal efectiva. El nutricionista por su parte, se enfrenta a un entramado de información que no es fácil resolver ya que el paciente se alimenta por tradición, por instinto, con lo que está disponible y con lo que más le gusta, entre muchas razones; pero casi siempre con escaso conocimiento científico, dando como resultado un desbalance entre sus necesidades, preferencias o posibilidades de alimentarse.

Esto representa un gran reto de comunicación: los sentires del doliente con los saberes del médico y nutricionista, inmersos en un ambiente hospitalario, comunitario y social. Este reto aparece desde el siglo V a.C. en el Corpus Hipocraticum donde los temas centrales fueron la relación del médico con el enfermo (visión ética y clínica), la terapia de intervención: remedios, incisiones, cauterizaciones, y la terapia dietética que incluía alimentación, actividad física, vestido, etc. La dietética se vuelve, entonces, protagonista del cuidado de la salud y de la enfermedad.
En la lectura de esta obra, se hace evidente la búsqueda apasionada de explicaciones de lo que le ocurre al enfermo. Se lo escucha y observa con ánimo de generar explicaciones y propuestas que lleve a solucionar el problema. Así, al paso de los años, las diferentes generaciones de médicos fueron haciendo propuestas de estudio de los enfermos influenciados por las diversas corrientes filosóficas, lingüistas y científicas.

El primer encuentro, que es verbal, el enfermo expresa su experiencia del mal que le aqueja, sus causas, sus fantasías o sus propuestas, el cual conocemos como interrogatorio o anamnesis (del

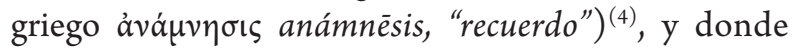
es analizado el síntoma: experiencia subjetiva e histórica de la enfermedad que el enfermo interpreta. El segundo encuentro ocurre a través de los órganos de los sentidos del explorador (inspección, palpación, percusión y auscultación) que pretende comprobar y confrontar el discurso del enfermo con hechos objetivos, que le permitan proponer explicaciones adecuadas de lo que está pasando. Esto es lo que recibe el nombre de signos clínicos.

El proceso que ocurre luego en la mente del clínico es fantástico. Como resultado del análisis de la información se concibe un nuevo constructo que es el síndrome (conjunto de signos y síntomas que puede explicar fisiopatológicamente el problema) y corresponde a la primera hipótesis generada a partir de los estudios paraclínicos (laboratorio y gabinete) que ayudarán a llegar al diagnóstico. En el área de nutrición hay varios ejemplos como el síndrome de caquexia-anorexia, la desnutrición, síndrome consuntivo, síndrome de mala absorción, etc. Del análisis del síndrome surge la propuesta diagnóstica.

El diagnóstico es fundamental para una adecuada tera-

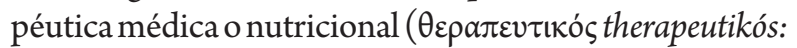
conjunto de prácticas y conocimientos encaminados al tratamiento de las dolencias) $)^{(4,5)}$. Este proceso es el que justifica la existencia de Nutrición o Nutriología Clínica. No es solamente dar de comer a los enfermos, ni llenar una serie de formularios para obtener una calificación, ni seguir algoritmos. Es conjuntar la ciencia, la evidencia, con la vivencia y entenderlas; ayudar a la armonía homeostática del enfermo. Acompañarlo, ver su evolución y sobre todo evaluar resultados en el ámbito individual y colectivo para después compartirlo con la comunidad científica.

En la presente revisión mostraremos algunos ejemplos de esta semiología de la nutrición, pero se invita 
al clínico y nutricionista a revisar los textos clásicos de Semiología y Propedéutica Médica, que seguramente ampliarán el conocimiento.

\section{SEMIOLOGÍA DEL APETITO Y SACIEDAD}

Desde el Corpus Hipocrático hay descripciones clínicas de la malnutrición, pero en sobrevivientes de la segunda guerra mundial se tienen mejores observaciones, más ordenadas de los cambios en la desnutrición crónica, evaluación del estado nutricional, más adelante, del síndrome de realimentación, así como los cambios del apetito y saciedad como importantes indicadores y predictores del estado nutricional ${ }^{(5)}$. En la Tabla $1 \mathrm{se}$ presentan algunas definiciones que más utilidad tienen en clínica.

García-Flores ${ }^{(6)}$ destaca cuatro factores importantes en la regulación de la ingesta a saber: a. socioculturales (estilo de vida y socioeconómicos), b. físicos o ambientales (apariencia, tamaño de la porción, etc.), c. biológicos (hormonas, contenido energético, cantidad, etc.) y d. psicológicos (expectativas, creencias, etc.), que deben interrogarse y considerarse para identificar las áreas de oportunidad de atención y riesgos del enfermo. Habrá que hacerlo con claridad en el lenguaje utilizado y estar seguro de que se ha comprendido bien principalmente en cuanto al tipo de alimentos, bebidas, cantidades, volúmenes, lugar donde se hacen los alimentos y tiempo para alimentarse, entre otros. Los factores sensoriales como el sabor, visión, textura, palatabilidad, olfato y audición son los determinantes del inicio y final del acto de comer. Sin embargo, las condiciones situacionales (lugar donde se alimenta, con quien, tiempo para alimentarse, etc.) y las psicológicas se interactúan para que se inicie o termine el acto de comer y la satisfacción del sujeto ${ }^{(6,7)}$.

Por otra parte, la ausencia de apetito como lo es la anorexia se define como la inapetencia, las faltas de

Tabla 1. Términos relacionados con el apetito y saciedad

\begin{tabular}{|c|c|}
\hline Término & Definición \\
\hline Hambre & $\begin{array}{l}\text { Sensación fisiológica o psicológica que induce a comer. Puede ser considerada un mecanismo de defensa que } \\
\text { alerta al organismo cuando existe una necesidad de nutrientes. En muchas ocasiones es instintiva. }\end{array}$ \\
\hline $\begin{array}{l}\text { Hambre } \\
\text { fisiológica }\end{array}$ & $\begin{array}{l}\text { En el hambre fisiológica el sujeto busca cualquier alimento "para llenar el estómago", es impulsiva y busca evitar } \\
\text { la hipoglucemia. Se acompaña de cólico abdominal inespecífico, cefalea, mareos, debilidad e incluso lipotimia. Es } \\
\text { secundaria a ayuno prolongado de más de } 6 \text { a } 8 \text { horas que puede ser por hábitos alimentarios deficientes, alguna } \\
\text { indicación médica o carencia de alimentos, mala planeación de horarios laborales o escolares. }\end{array}$ \\
\hline $\begin{array}{l}\text { Hambre } \\
\text { emocional }\end{array}$ & $\begin{array}{l}\text { El hambre emocional es desencadenada por el ocio, soledad, necesidad de afecto y trastornos de ansiedad, dando } \\
\text { satisfacción de corta duración y es causa frecuente de obesidad y trastornos en la conducta alimentaria. Se hará necesaria } \\
\text { la valoración psicológica adecuada ya que este factor puede ser determinante en el éxito de la atención nutricional. }\end{array}$ \\
\hline Saciación & $\begin{array}{l}\text { Es el momento en que se suspende el acto de comer por desaparición del hambre y que aparece aproximadamente } \\
\text { a los } 20 \text { minutos de haber iniciado la ingesta de alimentos. }\end{array}$ \\
\hline Saciedad & $\begin{array}{l}\text { Es el tiempo entre la saciación y la sensación nuevamente de hambre que puede tener una duración de } 6 \text { a } 8 \text { horas, } \\
\text { incluyendo el posprandial. }\end{array}$ \\
\hline Apetito & $\begin{array}{l}\text { Apetito es el deseo de ingerir alimentos como experiencia placentera a diferencia del hambre en donde la } \\
\text { sensación es desagradable e imperiosa de comer }{ }^{(6)} \text {. Puede ser de origen metabólico o psicológico. }\end{array}$ \\
\hline Anorexia & Es la ausencia de apetito que puede ser de origen orgánico o psicológico. No es falta de hambre. \\
\hline Hiporexia & Disminución del apetito. \\
\hline Hiperorexia & Incremento en el apetito. \\
\hline Hiperfagia & $\begin{array}{l}\text { Aumento en la ingesta de alimentos, independientemente del apetito o del hambre como se observa en la diabetes } \\
\text { mellitus o la bulimia. }\end{array}$ \\
\hline Polifagia & Ingesta frecuente de alimentos, independientemente de la cantidad o sensación de hambre. \\
\hline Palatabilidad & Cualidad de un alimento de ser grato al paladar. \\
\hline
\end{tabular}


ganas de comer, que frecuentemente es de origen psicológico, pero puede ser secundario a la acción del factor de necrosis tumoral alfa por su efecto anoxigénico común en enfermos con cáncer y enfermedades crónicas. El estudio metódico de inicio, evolución, variaciones y cantidades ingeridas determinará el riesgo de desnutrición, sus consecuencias y opciones terapéuticas. Hay que evaluar también si el paciente ha iniciado tratamientos previos para mejorar el apetito (orexigénicos) como el uso de cannabinoides, esteroides, etc. que en algunos pacientes tienen buenos resultados, pero que requiere una mayor evaluación ${ }^{(8)}$.

Desde 2003, la European Society for Clinical Nutrition and Metabolism (ESPEN) ha lanzado una fuerte campaña contra la desnutrición principalmente en hospitales y residencias de enfermos. En 2006 se hace una gran convocatoria conocida como Nutrition Day ${ }^{(9)}$, un estudio multicéntrico observacional realizado a través de una encuesta ${ }^{(10)}$, cuyo objetivo es mejorar los conocimientos sobre el cuidado nutricional en las unidades o servicios hospitalarios e implementar una atención nutricional adecuada. La encuesta permite un acercamiento apropiado al enfermo para detectar problemas con su alimentación en el último año y el momento actual, los factores y riesgos que pueden influir en desarrollar desnutrición.

Recientemente ESPEN (2019) publica criterios para el diagnóstico de la desnutrición (GLIM), proponiendo tres criterios fenotípicos (pérdida de peso no propositiva, disminución de la masa muscular y disminución del índice de masa corporal), y dos criterios etiológicos (disminución de la ingesta de alimento y gravedad de la inflamación asociada a la enfermedad). La suma de un criterio fenotípico y etiológico ayudan a hacer el diagnóstico ${ }^{(11)}$. La propuesta GLIM utiliza en forma inadecuada el término de diagnóstico al enunciar solo un signo y un síntoma, sin observar la semiología completa para elaborar una propuesta sindromática, y al final concluir que el término diagnóstico completo solo se utilizará después de la evaluación médico-nutricional completa.

No basta la anamnesis para un adecuado diagnóstico nutricional y es ahí donde la exploración física exhaustiva, en conjunto con el médico permitirá evaluar los signos clínicos que se pueden expresar en la piel, cavidad oral y composición corporal, y estudios paraclíni$\cos ^{(12)}$. La hipofunción y la atrofia son evidentes con síntomas como debilidad, cansancio, letargo, falta de concentración, aumento de la susceptibilidad a infecciones, etc., pero también con signos de disminución de la masa y fuerza muscular, marcha lenta, trastornos de la motilidad intestinal, fragilidad y caída del cabello, la descamación y delgadez de la piel. Los cambios en la cavidad oral por deficiencias vitamínicas como la lengua magenta, queilosis, glositis y gingivorragias, por mencionar. La exploración de la masa muscular como es la atrofia de músculos temporales, maseteros (Figuras 1 y 2), deltoides, bíceps, cuádriceps y gemelos, por mencionar los más frecuentes, y la exploración de la masa grasa a través de la grasa adiposa de Bichat en la mejilla, la grasa supraclavicular, la grasa intercostal, ayudado con la medición de los pliegues cutáneos, la medición de peso y talla para obtener el índice de masa corporal $^{(13,14)}$. El uso de estudios paraclínicos de composición corporal como la impedancia bioeléctrica, la doble absorbancia de Rayos X, el ultrasonido muscular y la dinamometría, ayudan a tener más datos medibles y comparables con la evolución del enfermo. Los estudios complementarios de laboratorio como la medición de albúmina, colesterol, biometría hemática, etc. completan la exploración bioquímica. La dilución principalmente se observa en la aparición de edema blando, que se desplaza con facilidad hacia las partes declives, no doloroso y que se ha descrito principalmente en el Kwashiorkor infantil y en los adultos en estado crítico.

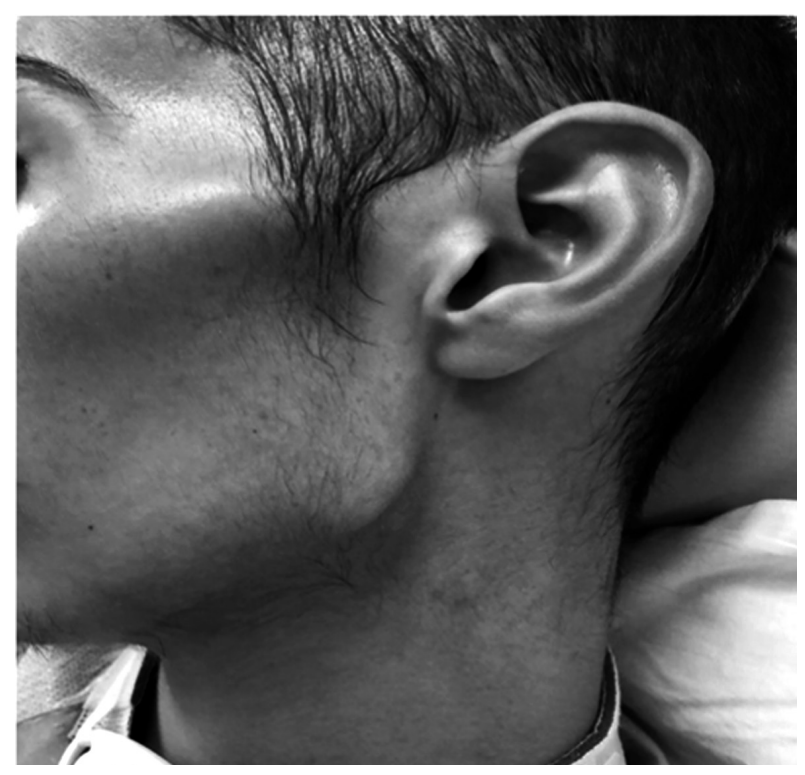

Figura 1. Atrofia muscular severa de músculos de la cara. Foto original.

\section{SEMIOLOGÍA DE LA MOTILIDAD INTESTINAL}

Un reto clínico para el médico y nutricionista es el paciente con trastornos de la motilidad intestinal ya 
que su origen es multifactorial y su presentación clínica puede ser de múltiples formas desde alteraciones en la boca, dolor abdominal, alteraciones en los hábitos de defecación, meteorismo, flatulencia, etc. Uno de los factores que determinan estos problemas es el desequilibrio en el microbiota intestinal conocido como disbiosis. La semiología de estos síntomas y signos gastrointestinales ${ }^{(15)}$ puede orientar al clínico en el reconocimiento de microorganismos causales de cada problema; y por tanto, apoyar la terapéutica y prevenir el deterioro del estado nutricional ${ }^{(16,17)}$. En la Tabla 2 se puede observar la relación de síntomas gastrointestinales y su asociación con la disbiosis observada.

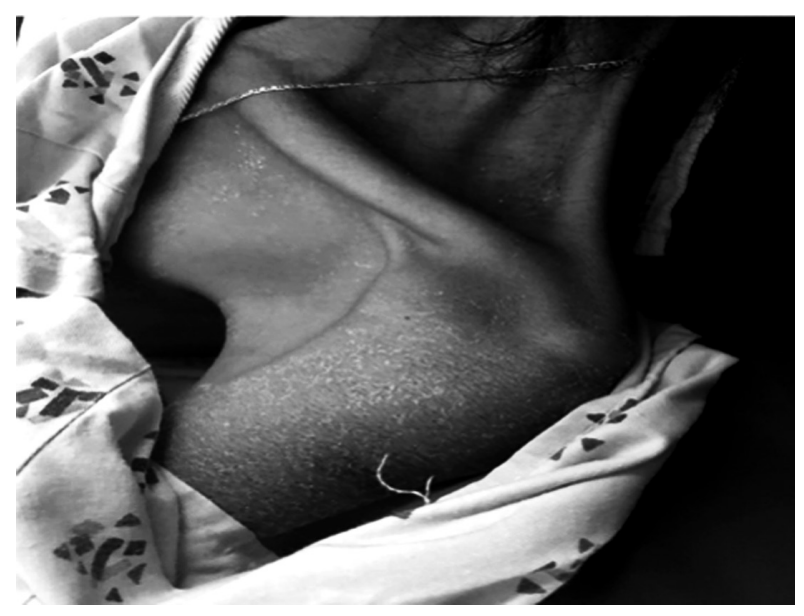

Figura 2. Atrofia muscular severa de músculos del hombro. Foto original

El 90 \% de los casos de halitosis se relacionan con caries, gingivo estomatitis, xerostomía, enterocolitis crónica por sobrecrecimiento bacteriano, y a la ingesta de cárnicos aromáticos, además que pueden asociarse con deficiencia vitamínicas del complejo B y vitamina $\mathrm{C}^{(14)}$. La náusea es el resultado de movimientos no coordinados de los músculos de la respiración, diafragma y abdomen. En ayuno, se asocia a gastritis o parasitosis (Ascaris) y en el posprandio a gastroparesia (hipotonía gástrica). De la náusea hay que saber su inicio, factores desencadenantes, factores que la disminuyen y si se acompaña de vómito. Esta situación debe ser analizada puesto que puede limitar la alimentación en forma significativa como ocurre con frecuencia en la quimioterapia. La pirosis es una sensación de ardor retroesternal recurrente, secundaria al reflujo gástrico por alteración de la actividad neuromuscular del esófago que ocasiona ondas peristálticas ascendentes. Esta sensación condiciona la ingesta de alimentos que favorecen la secreción gástrica como son los alimentos calientes, en exceso condimentados, y la ingesta de frutos cítricos.

El vómito es una reacción refleja, siempre precedida de náuseas y arqueo que busca lograr el vaciamiento gástrico. Al igual que la náusea hay que precisar el inicio, causas que lo propician, causas que lo disminuyen y fenómenos que lo acompañan. Es uno de los síntomas/ signos que mayor afectación tiene para el estado nutricional principalmente en enfermedades crónicas como el cáncer en donde se asocia a saciación temprana o "síndrome de comida pequeña". Hay que diferenciarlo de la regurgitación que puede verse en la hernia hiatal o en trastornos neurológicos crónicos y de la rumiación que se observa en enfermos psiquiátricos ${ }^{(14)}$.

El vómito en ayuno se debe a hipersecreción gástrica (como en el caso de la úlcera gastroduodenal) y el vómito posprandial se asocia a hipotonía gástrica ${ }^{(14)}$. La gastroparesia se acompaña de náuseas, vómito de retención (gran volumen de alimentos fermentados), saciedad temprana y sensación de plenitud, y se ha asociado a pérdida intersticial de células de Cajal y de nervios entéricos, así como a fibrosis estromal, cambios que son atribuidos a la pérdida de los macrófagos anti-inflamatorios heme-oxigenasa 1 positivo ${ }^{(18)}$ y se observa con mayor frecuencia en pacientes diabéticos y con enfermedades neurológicas crónicas. Es importante su diagnóstico oportuno ya que con frecuencia la alimentación por sonda es gástrica y puede propiciar la regurgitación en un estómago parético. Además, se puede agravar con la ingesta de alimentos ricos en grasas que disminuyen el vaciamiento gástrico. Los lipopolisacáridos y el ácido lipoteicoico (LTA), componentes de membrana de bacterias gram positivas (Firmicutes), también se asocian a daño de neuronas entéricas (al unirse a TLR4 y TLR2 respectivamente), al incrementar el estrés oxidativo y la inflamación mediada por TNT-a ${ }^{(19)}$.

La epigastralgia en ayuno se relaciona con hipersecreción gástrica (úlcera duodenal o pilórica), y la posprandial con úlcera gástrica, antritis y a dispepsias reflejas (biliar, pancreática, apendicular, ovárica ${ }^{(14)}$. La semiología del dolor por úlcera gástrica o duodenal son diferentes y limitan en forma importante la ingesta de alimentos productores de ácido y de grasas, además que hay que agregar preguntas de disminución de la ingesta de alimentos o pérdida de peso que sugerirían un problema neoplásico que requerirá una Terapia Nutricional Especializada temprana con el fin de prevenir complicaciones. Con frecuencia estos pacientes tienen desorden en sus horarios de alimentación y con periodos de ayuno prolongados, además de comidas 
Tabla 2. Signos y síntomas asociados a la disbiosis

\begin{tabular}{|l|l|}
\hline \multicolumn{1}{|c|}{ Signos y síntomas (causa orgánica) } & \multicolumn{1}{c|}{ Disbiosis observada } \\
\hline Halitosis (caries, gingivoestomatitis) & Sobrecrecimiento bacteriano \\
\hline Náusea (gastritis, hipotonía) & Áscaris \\
\hline Reflujo gastroesofágico (peristalsis ascendente) & Helicobacter pylori \\
\hline Dispepsia distónica (duodenitis eosinofílica) & $\begin{array}{l}\text { Mayor: Helicobacer pylori, Streptococus } \\
\text { Menor: Prevotella, Veillonela, Actinomyces }\end{array}$ \\
\hline Vómito (hipersecreción, hipotonía gástrica) & Sobrecrecimiento bacteriano \\
\hline Gastroparesia (pérdida de macrófagos hemOx1) & Mayor: Firmicutes gran - positivas \\
\hline $\begin{array}{l}\text { Estreñimiento funcional (ausencia excitabilidad } \\
\text { nerviosa) }\end{array}$ & $\begin{array}{l}\text { Mayor: Bacteroidetes (mucoso), E. coli, S. aureus, } \\
\text { Menor: Bifidobacteria, Bacteroides, Prevotella }\end{array}$ \\
\hline Diarrea (tránsito acelerado) & Menor: Porphyromonas, Prevotella y Fusobacterium, menor diversidad \\
\hline Cólico abdominal & Menor: Lactobacillus y Bifidobacterium \\
\hline Distensión abdominal (neurógena) & Menor diversidad y menor riqueza filogénica \\
\hline Meteorismo (neurodistonia, hiperfermentación) & Mayor: Methanobacteriales y Clostridiales \\
\hline Dolor abdominal (Epigastralgia, SII, Ell) & $\begin{array}{l}\text { Mayor: PAMPS, Shigella, E.coli, Salmonella, Campylobacter jejuni, Norovirus, } \\
\text { Giardia duodenalis y Blastocystis } \\
\text { Menor: Bifidobacterium, Bacteroidetes, Bacteroides y Prevotella }\end{array}$ \\
\hline
\end{tabular}

condimentadas. Todos estos factores modifican el sentido del gusto, la calidad del bolo alimenticio, del apetito y de la saciación.

Por otra parte, la dispepsia funcional o distónica, con frecuencia confundida con el Síndrome de Colon Irritable, tiene criterios bien establecidos: Roma IV ${ }^{(20)}$ como la presencia durante 3 meses de plenitud posprandial, saciedad temprana, dolor, ardor epigástrico, que pueden acompañarse de náusea, eructos excesivos o distensión ${ }^{(21)}$. Relacionada a menudo con episodios de tensión emocional, hace que el paciente modifique de manera importante sus hábitos de alimentación, lo que empeora los síntomas, disminuye el peso y favorece la automedicación. Esta entidad se explica por disfunción motora, inflamación de bajo grado (duodenitis eosinofílica), reducción de la acidez gástrica y daño de la mucosa duodenal (incremento de la permeabilidad) (21), factores que han sido asociados a infección crónica por Helicobacter pylori ${ }^{(22)}$, misma que se acompaña de incremento de Streptococus, reducción de Prevotella, Veillonela y Actinomyces, incremento de la carga bacteriana y reducción de la diversidad ${ }^{(21,23)}$.

La reducción de la diversidad bacteriana e incremento de la permeabilidad del intestino delgado, se asocian a los síntomas de los desórdenes funcionales
GI, como son diarrea, dolor y distensión abdominal, particularmente posterior a la ingesta de antibióticos, de inhibidores de bomba de protones, en mayores de 50 años y con historia de cirugía intestinal ${ }^{(24)}$. La distensión abdominal neurógena, también conocida como seudociesis, está acompañada del signo de "patadas" (por contracciones de la pared abdominal), sin emisión de gases por boca ni ano ${ }^{(14)}$. Aspirados duodenales en pacientes sintomáticos revelan menor cantidad de Porphyromonas, Prevotella y Fusobacterium, menor diversidad y riqueza filogénica, y la expresión funcional (vías relacionadas con el estrés oxidativo y metabolismo de azúcares simples), regulada por la ingesta, caracteriza la disbiosis, más que el sobrecrecimiento bacteriano per se $e^{(24)}$.

El dolor abdominal inespecífico característico de la enfermedad intestinal inflamatoria (EII), del síndrome de intestino irritable (SII), del cólico infantil, de la dispepsia funcional y de la cistitis intersticial, se asocia al estímulo, directo o indirecto (por liberación de citocinas y reclutamiento de neutrófilos) de las neuronas del ganglio dorsal. Este estímulo es mediado por los receptores TLR4, TLR2, TLR5 y Dectina-1, y proviene de los patrones moleculares asociados a patógenos (PAMPS) como son: LPS, LTA, peptidoglicanos, b-glicanos, pép- 
tidos formilados de $S$. aureus, la toxina a-hemolisina. Flagelina, glucanos, mananos, chitinas ${ }^{(16,17)}$. Es un dolor tipo espasmódico, crónico con exacerbaciones intermitentes, en el trayecto de marco cólico sin una causa específica que lo aumente y puede calmar con la defecación y la flatulencia secundaria a los gases generados por la disbiosis. La administración temprana de antibióticos se asocia a hipersensibilidad visceral en el adulto, y el dolor y la inflamación se reducen con la administración de probióticos (Lactobacillus reuteri, acidofilus, paracasei y ramnosus GG, Bifidobacterias infantis, breve, Roseburia hominis, F. frausnitzii y E. coli Nissle) que estimulan la síntesis de GABA, serotonina y de butirato, e inhiben el NF-kb ${ }^{(16,17)}$.

El SII se manifiesta con dolor abdominal, estreñimiento, diarrea, distensión y meteorismo, conocido tradicionalmente con síndrome disenteriforme y que se confunde con parasitosis intestinal como la amibiasis y el paciente recibe con frecuencia antimicrobianos que agravan el problema. Su fisiopatología se ha asociado a alteraciones en el eje microbioma - intestino - cerebro, en el sistema inmune (inflamación en pared intestinal), a cambios en la permeabilidad y a disbiosis ${ }^{(23)}$. En los pacientes con SII con diarrea, se observa una mayor concentración sérica (pero no fecal) de propionato y de butirato ${ }^{(25)}$. El microbiota en el SII se caracteriza por disminución de Bifidobacterium, Bacteroidetes, Bacteroides y Prevotella, así como incremento de Firmicutes, Clostridium, Ruminococcus y Roseburia ${ }^{(25)}$. El dolor por SII postinfección se ha asociado a incremento de Shigella, E. coli, Salmonella, Campylobacter jejuni, Norovirus, Giardia duodenalis y Blastocystis ${ }^{(16)}$. Al corregir el equilibrio del microbioma y los hábitos de alimentación con frecuencia se obtiene buena respuesta. El abuso de antiespasmódicos, antiflatulentos y antiácidos no resuelven el problema y retardan el diagnóstico.

La diarrea es un incremento en el número de evacuaciones al día y con disminución de la consistencia de diversa etiología, debe analizarse más bien como un verdadero síndrome que puede alterar el estado de hidratación, el estado nutricional y se acompaña de importantes cambios en el microbioma intestinal, con inflamación aguda o crónica. Su estudio semiológico preciso está fuera del alcance del presente documento. Las principales causas pueden ser bacterianas o virales a investigar enteritis estafilocócica, parasitosis, colon irritable (la palpación de colon sigmoideo es diagnóstica) y procesos neurógenos como causas asociadas a la disbiosis. Las matutinas se asocian a colon irritable y las nocturnas a parasitosis ${ }^{(14)}$.
El estreñimiento es un trastorno de la defecación con pausas de más de 48 - 72 horas entre las deposiciones. Las principales causas son una alimentación baja en residuo (la cual no permite la adecuada excitabilidad de los centros nerviosos que regulan la peristalsis), la poca hidratación, el sedentarismo, las distonías neurovegetativas del colon ${ }^{(14)}$ y la reducción en la concentración de 5-HT (por regulación alta de su recaptura) ${ }^{(26)}$. La retención fecal en colon derecho, conocida como toxemia esterocoracea, se acompaña de anorexia, halitosis, lengua saburral, insomnio, irritabilidad, vértigo, mareo, fiebre, depresión y ansiedad y puede alterar el estado nutricional ${ }^{(14)}$. El estreñimiento funcional se ha asociado a menor cantidad de Actinobacteria (Bifidobacteria), Lactobacillus y Bacteroides, mayor cantidad de E. coli y $S$. aureus en heces de adultos y mayor nivel de Bacteroidetes a nivel mucoso ${ }^{(24)}$. En los pacientes con EII con constipación, se observa mayor Veillonella y menor Clostridium cocoides, Actinobacteria en muestras fecales, e incremento de Bacteroidetes a nivel mucoso ${ }^{(24)}$.

El meteorismo consiste en la distensión del abdomen por gases contenidos a nivel gastrointestinal (percusión timpánica), cuyas causas incluyen aerofagia, exceso de fermentación, neurodistonía (evita resorción de gases), paresia intestinal, hipocalemia, ingesta de alimentos productores de gas, síndrome premenstrual y parasitosis ${ }^{(13)}$. La reducción del tránsito intestinal y el meteorismo se asocian a incremento de Methanobacteriales y Clostridiales ${ }^{(16)}$. La utilización de probióticos (Bifidobacterium lactis y longum, y Lactobacillus paracasei, rhamnosus y plantarum) ha sido efectiva en estos pacientes ${ }^{(24)}$. Cabe recalcar que la dieta es una variable de confusión a considerar dado que tiene un impacto inmediato en la composición del microbioma ${ }^{(25)}$. Las exacerbaciones de la EII se han relacionado con incremento de Proteobacteria, Candida albicans y Basidiomycota/Ascomycota, así como a menor cantidad de Clostridium cocoides, F. prausnitzii y Bifidobacterium, y Saccharomyces cerevisiae ${ }^{(16)}$.

El cólico abdominal es un dolor de comienzo brusco, de presentación intermitente, que es aliviado por la posición fetal, se acompaña de borborigmos y del deseo de defecar, así como de bradicardia vagotónica ${ }^{(14)}$. El cólico infantil se caracteriza por menor riqueza bacteriana, reducción de Lactobacillus y Bifidobacterium e incremento de gram negativas ${ }^{(16)}$. En la diverticulosis colónica se observa depleción de Clostridium IV, IX, Fusobacterium y Lactobacillaceae ${ }^{(16)}$.

En la práctica clínica, un método alternativo para realizar el diagnóstico de disbiosis es a través de la 
semiología de los desórdenes funcionales intestinales y del diagnóstico terapéutico, con el empleo, basado en evidencia, de probióticos ${ }^{(22)}$ y modificaciones en la dieta (ejemplo: dieta baja en FODMAP) ${ }^{(16)}$.

\section{CONCLUSIONES}

En la actualidad, el utilitarismo ha permeado todas las áreas y la medicina no es la excepción. El exceso de tecnología y medios de información ha creado la moderna torre de babel del conocimiento, deteriorando el humanismo que ha caracterizado históricamente al acto médico. El encuentro del enfermo con el asistente en salud (médico, nutricionista y enfermera) es el fundamento de la atención en donde un buen estudio semiológico y propedéutico del paciente puede garantizar un adecuado método científico que lleva primero a una identificación de riesgos, después a un diagnóstico, para culminar en una adecuada meta y plan terapéutico-nutricional, evaluable en forma continua para la comprobación de resultados. Volver a la educación clínica es una excelente alternativa, siempre que se adecúen los estudios paraclínicos (laboratorio y gabinete) como los modernos signos bioquímicos y de imagen para integrar mejores y más precisos diagnósticos.

\section{Declaración de autoría}

Todos los autores participaron en la recopilación de la información. IC y JC participaron en la primera parte del abordaje semiológico, $\mathrm{AD}$ en la sección de apetito y saciedad y MB en la sección de la semiología de la función intestinal y microbiota. Una vez recopilada la información le correspondió a JC el orden y redacción final. Todos los autores revisaron el manuscrito y validaron su versión final.

\section{Declaración de conflicto de intereses}

No hay conflicto de intereses.

\section{Financiamiento}

Ninguno.

\section{Referencias bibliográficas}

1. Samuel VT, Shulman GI. Integrating Mechanisms for insulin Resistance: Common Threads and Missing Link. Cell. 2012; 148(5); 852-71. doi: https://doi.org/10.1016/j. cell.2012.02.017.
2. Ceccotti EL, Sforza RR, Carzoglio FJC, Luberti R, Flichman JC. El diagnóstico en clínica estomatológica. $1^{\text {a }}$ edición. Buenos Aires, Argentina: Editorial Médica Panamericana; 2007.

3. Goic GA. Sobre el origen y desarrollo del libro de Semilogía Médica. Rev.Med. Chile. 2018; 146(3): 387-90. doi: http:// dx.doi.org/10.4067/s0034-98872018000300387.

4. Real Academia Española. Diccionario de la lengua española [Internet] 23 edición 2014. [Fecha de consulta: 30 de mayo de 2020]. Disponible en: https://www.rae.es/sites/default/ files/Dossier_Prensa_Drae_2014_5as.pdf.

5. Argente HA, Alvarez ME. Semiología Médica: Fisiopatología, Semiotécnica y Propedéutica. Enseñanza basada en el paciente. $1^{\mathrm{a}}$ edición. España: Editorial Médica Panamericana; 2005.

6. García-Flores CL, Martínez MAG, Beltrán MCP, ZepedaSalvador AP, Solano SLV. Saciación vs saciedad: reguladores del consumo alimentario. Rev.Med.Chile. 2017;145(9): 1172-8. doi: http://dx.doi.org/10.4067/s0034-98872017000901172.

7. Zijltra N, Mars M, Stafleu A, De Graaf C. The effect of texture differences on satiation in 3 pair of solid food. Appetite. 2010;55(3):490-7. doi: https://doi.org/10.1016/j. appet.2010.08.014.

8. Bellocchio L, Lafenêtre P, Cannich A, Cota D, Puente N, Grandes P, et al. Bimodal control of stimulated food intake by the endocannabinoid system. Nat Neurosci. 2010;13(3):2813. doi: https://doi.org/10.1038/nn.2494.

9. Nutrition Day Worlwide [Internet]. [Fecha de consulta: 28 de mayo de 2020. Disponible en: https://www.espen.org/ nutritionday-worldwide.

10. Heismayr M, Schindler K, Simon J, Kiss N, Kosak S, Bauer P, et al. Nutrition Day Worldwide. Hoja del paciente [Internet]. Versión 1; 07-10-2016 [Fecha de consulta: 28 de mayo de 2020] Disponible en: https://www.nutritionday.org/cms/ upload/pdf/1_for_hospitals/1.3.participate/Spanish/ND_ sheet3_spanish_geschwrzt.pdf.

11. Cederhol T, Jensen GL, Correia MITD, Gonzalez MC, Fukushima R, Higashiguchi T, et al. GLIM criteria for the diagnosis of malnutrition - A consensus report from the global clinical nutrition community. Clin Nutr. 2019; 38(1):1-9. doi: https://doi.org/10.1016/j.clnu.2018.08.002.

12. Touger-Decker R, Mobley C, Academy of Nutrition and Dietetic. Position of the Academy of Nutrition and Dietetics: Oral health and Nutrition. J Acad Nutr Diet. 2013;113(5):693- 701. doi: https://doi.org/10.1016/j. jand.2013.03.001.

13. Martínez CGT, García- Aranda JA. Desnutrición energéticoproteica. En:Kaufer-Howwirtz M, Pérez-Lizaur AB, Arroyo P. Nutriología médica. $4^{\text {a }}$ edición. México: Editorial Médica Panamericana; 2015. p. 1-34.

14. Surós BA, Surós BJ. Gastroenterología, Hepatología. En: Semiología Médica y Técnica Exploratoria. Octava Edición. España: Editorial Elsevier Masson, 2001 p.357-495. 
15. Cryan JF, O'Riordan K, Cowan SMC, Sandhu KV, Bastiaanssen TFS, Boehme M, et al. The Microbiota - GutBrain Axis. Physiol Rev. 2019;99(4):1877-2013. doi: https:// doi.org/10.1152/physrev.00018.2018.

16. Defaye M, Gervason S, Altier C, Berthon JY, Ardid D, Filaire E, et al. Microbiota: a novel regulator of pain. J Neural Transm. 2020; 127(4): 445-65. doi: https://doi.org/10.1007/s00702019-02083-z.

17. Guo R, Chen LH, Xing C, Liu T. Pain regulation by gut microbiota: Molecular mechanisms and therapeutic potential. Br J Anaesth. 2019;123(5):637-54. doi: https://doi. org/10.1016/j.bja.2019.07.026.

18. Bekkelund M, Sangnes DA, Gunnar HJ, Aabakken L. Pathophysiology of idiopathic gastroparesis and implications for therapy. Scand J Gastroenterol. 2019;54(1):8-17. doi: https://doi.org/10.1080/00365521.2018.1558280.

19. Nyavor Y, Brands CR, May G, Kuther S, Nicholson J, Tiger $\mathrm{K}$, et al. High-fat diet-induced alterations to gut microbiota and gut-derived lipoteichoic acid contributes to the development of enteric neuropathy. Neurogastroenterol Motil. 2020;32(7):e13838. doi: https://doi.org/10.1111/ nmo.13838.

20. Sebastian DJJ. Los nuevos criterios de Roma (IV) de los Trastornos Funcionales Digestivos en la práctica clínica. Med Clin. 2017;148(10):464-8. Doi: https://doi.org/10.1016/j. medcli.2016.12.020.
21. Tack J, Carbone F. Functional dyspepsia and gastroparesis. Curr Opin Gastroen. 2017; 33(6):, 446-54. doi: https://doi. org/10.1097/MOG.0000000000000393

22. Kim Y, Chung WC, Kim BW, Kim SS, Kim J, Kim JN, et al. Is Helicobacter pylori Associated Functional Dyspepsia Correlated With Dysbiosis? J Neurogastroenterol Motil. 2017;23(4):504-16. doi: https://doi.org/10.5056/ jnm17066.

23. Zhong L, Shanahan ER, Raj A, Koloski NA, Fletcher L, Morrison M, et al. Dyspepsia and the microbiome: time to focus on the small intestine. Gut. 2017; 66(6):1168-9. doi: https://doi.org/10.1136/gutjnl-2016-312574.

24. Ohkusa T, Koido S, Nishikawa Y, Sato N. Gut microbiota and Chronic Constipation: A Review and Update. Front Med. 2019;6:19. doi: https://doi.org/10.3389/fmed.2019.00019.

25. Enck $P$, Mazurak N. Dysbiosis in functional bowel disorders. Ann Nut Metab. 2018; 72(4): 296-306. doi: https://doi. org/10.1159/000488773

26. Cao H, Liu X, An Y, Zhou G, Liu Y, Xu M, et al. Dysbiosis contributes to chronic constipation development via regulation of serotonin transporter in the intestine. Sci Rep. 2017; 7(1):10322. doi: https://doi.org/10.1038/s41598-01710835-8. 